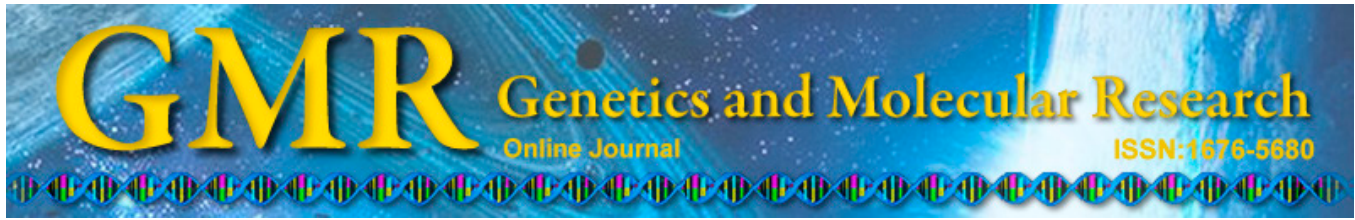

\title{
Effects of different anesthetic approaches on postoperative complications in HIV-infected patients
}

\author{
W.W. Zhang1, Y.P. Wang ${ }^{2}$, Y.Q. Wang ${ }^{1}$, X.M. Ji ${ }^{3}$ and M.Y. Zhang ${ }^{4}$ \\ ${ }^{1}$ Department of Anesthesia, Medical College Affiliated Hospital of Binzhou, \\ Shandong, China \\ ${ }^{2}$ Department of Electromyography, \\ Medical College Affiliated Hospital of Binzhou, Shandong, China \\ ${ }^{3}$ Department of Radiation Oncology, Beijing Chao Yang Hospital, \\ Capital Medical University, Beijing, China \\ ${ }^{4}$ Department of Anesthesia,Shangdong Province-owned Hospital, \\ Shandong, China \\ Corresponding author: M.Y. Zhang \\ E-mail: yaoqiwang@126.com
}

Genet. Mol. Res. 14 (3): 9130-9135 (2015)

Received November 10, 2014

Accepted March 11, 2015

Published August 7, 2015

DOI http://dx.doi.org/10.4238/2015.August.7.22

ABSTRACT. The aim of this study was to investigate the effects
of three different methods of anesthesia on patients with human
immunodeficiency virus (HIV) infection, which could lead to an
improvement in postoperative outcomes in these patients. A total of
90 patients undergoing an operation while being treated for an HIV
infection were included in this study. Patients were divided into three
groups ( $\mathrm{N}=30$ /group) based on the type of anesthesia administered:
general anesthesia, local anesthesia, and combined spinal epidural
anesthesia (CSEA). The effects of local infiltration of anesthesia
and peripheral nerve block local anesthesia were examined in the
local anesthesia group. The CSEA group examined the effects of
spinal anesthesia in HIV-infected patients. We compared the vital 
signs of the three groups during the preoperative period, at incision, and during the postoperative recovery period. The CSEA group had a significantly higher mean preoperative $\mathrm{CD}^{+} \mathrm{T}$ lymphocyte count compared with the general anesthesia and local anesthesia groups $(\mathrm{P}<0.05)$. We found that the three kinds of anesthesia methods administered to HIV-infected patients could be used with considerable safety and can be selected according to the clinical need and type of surgical procedure.

Key words: Anesthetic approach; HIV infection; Side effects; Surgery

\section{INTRODUCTION}

Human immunodeficiency virus (HIV)-infected patients have acquired immunodeficiency syndrome that was caused by infection with HIV. These patients have highly compromised immune systems and, thus, are at higher risk to fall ill. In addition, they have a low tolerance to injury (Lund et al., 2011; Klein et al., 2014; Redman et al., 2014). In recent years, these patients have undergone an increasing number of surgical procedures (Karpelowsky et al., 2011), and several anesthetic approaches have been studied previously (Evron et al., 2004; Bornard et al., 2011). At present, there are no special anesthetic drugs or methods to administer to HIV-infected patients (Krugova et al., 2010; Bajwa and Kulshrestha, 2013). Whether common methods, such as general anesthesia, local anesthesia, or combined spinal epidural anesthesia (CSEA) (Avidan et al., 2002), that were administered to HIV-infected patients caused different outcomes for patients without HIV-infection is still not well understood (Ayers et al., 1993). Here, we assessed 90 HIV-infected patients who were administered three different anesthetic approaches. We recorded the incidence of complications (Ferrero and Bentivoglio, 2003) and other indexes, such as changes in hemodynamics, during the perioperative period so that we could monitor the effect of these different anesthetic approaches on postoperative complications in HIV-infected patients.

\section{MATERIAL AND METHODS}

\section{General data}

A total of $90 \mathrm{HIV}$-infected patients underwent a surgical procedure in our hospital from January 2010 to January 2013. The 90 cases were divided into three groups according to anesthetic management: general anesthesia, local anesthesia, and CSEA. Each group contained 30 cases and the patient demographics were as follows: general anesthesia group (16 males and 14 females; mean age of $42.7 \pm 6.1$ years), and mean course of $3.7 \pm 1.1$ years; local anesthesia group (17 males and 13 females, mean age of $42.6 \pm 6.1$ years, and mean course of $3.6 \pm 1.1$ years); CSEA group (16 males and 14 females, mean age of $42.7 \pm 6.2$ years, and mean course of $3.8 \pm 1.1$ years). There were no significant differences of age, gender, and duration of disease between the three groups. The types of surgical procedures that HIVinfected patients underwent were: 5 spinal operations, 23 head-neck operations, 9 limb surgeries, 18 perianal operations, 14 thoracic-abdominal surgeries, 9 endoscopic procedures, and 
12 gynecologic operations. This study was conducted in accordance with the Declaration of Helsinki and with approval from the Ethics Committee of Medical College Affiliated Hospital of Binzhou. Written informed consent was obtained from all participants.

\section{Anesthesia}

All patients received an intramuscular injection of $0.5 \mathrm{mg}$ atropine and $0.1 \mathrm{~g}$ sodium phenobarbital $30 \mathrm{~min}$ before receiving anesthesia. In the operating room, patients were monitored by an electrocardiogram (Shenzhen Mindray Medical International, Co., Ltd., China). General anesthesia was administered via an Ohmeda 7000 (Datex-Ohmeda Company, USA). General anesthetic drugs contained: propofol (Fresenius Kabi, China), midazolam (JiangSu Enhua Pharmaceutical, Ltd., China), remifentanil and fentanyl (YiChang Renfu Pharmaceutical, Ltd.), sevoflurane (Shanghai Hengrui Pharmaceutical Co., Ltd.), and Cisatracurium (JiangSu Dongying Pharmaceutical, Ltd.). Local anesthetic drugs contained $0.375 \%$ ropivacaine (JiangSu Dongying Pharmaceutical, Ltd.) and 1\% lidocaine (Dalian Otsuka Pharmaceutical Co., Ltd.). The CSEA group included $0.5 \%$ ropivacaine and $0.5 \%$ bupivacaine (Shanghai Zhaohui Pharmaceutical, Ltd.).

\section{Observation indexes}

Heart rate (HR), peripheral capillary oxygen saturation $\left(\mathrm{SpO}_{2}\right)$, mean arterial pressure (MAP), anesthesia duration, and postoperative complications were observed at different time points, including before anesthesia, after induction, at skin incision, and during the postoperative recovery period for each of the three groups. A count of $\mathrm{CD}^{+} \mathrm{T}$ cells was measured by fluorescence-activated cell sorting Calibur FCM (Beckman Coulter, Ltd., China). All samples were stored in an $-80^{\circ} \mathrm{C}$ storage freezer (Haier Bio-Medical Ltd., China).

\section{Statistical analysis}

Statistical analysis was performed using the SPSS13.0 software. Measured data are reported as means $\pm \mathrm{SD}$. A Student $t$-test was used to compare data between groups, and a paired $t$-test was used to compare data in the same group. $\mathrm{CD}^{+} \mathrm{T}$ cell count data were compared using the chi-square test. A $\mathrm{P}$ value of $\mathrm{P}<0.05$ was considered a statistically significant difference.

\section{RESULTS}

\section{Clinical index}

Table 1. Comparison of clinical indices between the three anesthesia groups (means \pm SD).

\begin{tabular}{llccc}
\hline Group & $\mathrm{N}$ & $\mathrm{CD} 4^{+} \mathrm{T}$ cells count $(\mu \mathrm{L})$ & Anesthetic time $(\mathrm{min})$ & Weight $(\mathrm{kg})$ \\
\hline General anesthesia & 30 & $205.3 \pm 142.9$ & $178.2 \pm 124.8$ & $61.4 \pm 8.7$ \\
Local anesthesia & 30 & $196.2 \pm 142.4$ & $112.2 \pm 76.5^{*}$ & $63.3 \pm 6.8$ \\
Combined spinal-epidural anesthesia & 30 & $320.4 \pm 138.6^{* \#}$ & $135.4 \pm 60.9$ & $63.3 \pm 7.6$ \\
\hline
\end{tabular}

General anesthesia group $v s$ local anesthesia group, combined spinal epidural anesthesia group, ${ }^{*} \mathrm{P}<0.05$; combined spinal epidural anesthesia group vs local anesthesia group, ${ }^{\#} \mathrm{P}<0.05$. 
Analysis of perioperative $\mathrm{CD}^{+} \mathrm{T}$ cells counts revealed significantly higher levels in the CSEA group compared with the other groups $(\mathrm{P}<0.05)$. Additionally, the duration of local anesthesia was significantly shorter than that in the general anesthesia group $(\mathrm{P}<0.05$, Table 1$)$.

\section{Postoperative complications}

The incidence rate of complications in the general anesthesia, local anesthesia, and CSEA groups was $16.67,8.33$, and $15.00 \%$ respectively. The incidence rate of complications was significantly lower in local anesthesia group than that in the other groups $(\mathrm{P}<0.05$, Table 2$)$.

Table 2. Comparison of postoperative complications between the three anesthesia groups (\%).

\begin{tabular}{lccccc}
\hline Group & $\mathrm{N}$ & Fever & Grade B healing & Grade C healing & Total \\
\hline General anesthesia & 30 & $6(20.00)$ & $2(6.67)$ & $2(6.67)$ & $10(16.67)$ \\
Local anesthesia & 30 & $1(1.67)^{*}$ & $4(6.67)$ & $0(0.00)^{*}$ & $5(8.33)^{*}$ \\
Combined spinal-epidural anesthesia & 30 & $6(20.00)^{*}$ & $2(6.67)$ & $1(1.67)$ & $9(15.00)^{*}$ \\
\hline
\end{tabular}

General anesthesia group $v s$ local anesthesia group, combined spinal epidural anesthesia group, $* \mathrm{P}<0.05$; combined spinal epidural anesthesia group vs local anesthesia group, ${ }^{\#} \mathrm{P}<0.05$

\section{Vital signs}

Relative to baseline levels recorded before anesthesia administration, MAP decreased after induction and at the time of incision in the general anesthesia group, but $\mathrm{SpO}_{2}$ increased. In addition, in the local anesthesia group, HR increased after the onset of anesthesia, during incision, and during the postoperative period, and MAP increased during the postoperative period. In the CSEA group, HR significantly increased after anesthesia administration, but MAP declined significantly $(\mathrm{P}<0.05)$. Compared with the general anesthesia group, HR was faster, MAP increased, and $\mathrm{SpO}_{2}$ declined in the local anesthesia group. In addition, HR was faster after induction but slower during the postoperative period in the CSEA group compared with the general anesthesia group, and $\mathrm{SpO}_{2}$ decreased significantly in the CSEA group, compared with the general anesthesia group $(\mathrm{P}<0.05$, Table 3$)$.

Table 3. Comparisons of vital signs between the three anesthesia groups (means $\pm \mathrm{SD}$ ).

\begin{tabular}{|c|c|c|c|c|c|c|}
\hline Index & Group & $\mathrm{N}$ & Before anesthesia & After induction & During incision & Post-operation \\
\hline \multirow[t]{3}{*}{ HR (beats/min) } & General anesthesia & 30 & $81 \pm 16$ & $74 \pm 10$ & $76 \pm 11$ & $81 \pm 12$ \\
\hline & Local anesthesia & 30 & $78 \pm 11$ & $90 \pm 12^{* \Delta}$ & $91 \pm 9^{* \# \Delta}$ & $90 \pm 9^{* \# \Delta}$ \\
\hline & Combined spinal-epidural anesthesia & 30 & $80 \pm 17$ & $85 \pm 15^{* \Delta}$ & $75 \pm 12$ & $74 \pm 8^{*}$ \\
\hline \multirow[t]{3}{*}{ MAP (mmHg) } & General anesthesia & 30 & $90 \pm 12$ & $77 \pm 8^{\Delta}$ & $81 \pm 9^{\Delta}$ & $87 \pm 9$ \\
\hline & Local anesthesia & 30 & $86 \pm 11$ & $91 \pm 7$ *\# & $92 \pm 8^{* \#}$ & $93 \pm 9 * \# \Delta$ \\
\hline & Combined spinal-epidural anesthesia & 30 & $89 \pm 12$ & $79 \pm 9^{\Delta}$ & $81 \pm 9$ & $86 \pm 8$ \\
\hline \multirow{3}{*}{$\mathrm{SpO}_{2}(\%)$} & General anesthesia & 30 & $97 \pm 3$ & $100 \pm 2^{\Delta}$ & $101 \pm 1^{\Delta}$ & $102 \pm 1^{\Delta}$ \\
\hline & Local anesthesia & 30 & $97 \pm 2$ & $98 \pm 1 * \#$ & $98 \pm 1 * \#$ & $97 \pm 1$ *\# \\
\hline & Combined spinal epidural anesthesia & 30 & $96 \pm 1$ & $98 \pm 1^{*}$ & $98 \pm 1 *$ & $97 \pm 1^{*}$ \\
\hline
\end{tabular}

General anesthesia group $v s$ local anesthesia group, general anesthesia group $v s$ combined spinal epidural anesthesia group, ${ }^{*} \mathrm{P}<0.05$; combined spinal epidural anesthesia group $v s$ local anesthesia group, ${ }^{*} \mathrm{P}<0.05$; comparisons among groups, ${ }^{\Delta} \mathrm{P}<0.05$. 


\section{DISCUSSION}

HIV-infected patients are prone to contracting many diseases because of a compromised immune system, which causes a high incidence rate of physiological dysfunction and other adverse events peri operation period. Moreover, owing to the physical stress incurred from some surgeries, the sympathetic, adrenal medulla, hypothalamus, and hypophysis endocrine systems could become disordered (Madzimbamuto et al., 2013). Because anesthesia administration affects the aforementioned systems, the choice of anesthetic treatment is important. At present, the anesthesia regimen for patients with HIV infection still lacks an authoritative guide, and patient tolerance and the types of anesthesia medications used are the main problems faced by anesthesiologists. Moreover, proper anesthetic management can greatly alleviate the stress response (Karpelowsky et al., 2011), which can lead to improved postoperative outcomes for patients. This study showed that general anesthesia, spinal anesthesia, and peripheral nerve block could be used safely in patients with HIV infection.

Many factors decided the status of postoperative recovery, such as patient condition, surgical category, the incidence of perioperative complications, and anesthetic method selection (Bornard et al., 2011). However, general anesthesia can suppress immune function; thus, the safety of HIV-infected patients undergoing general anesthesia was a concern to both anesthesiologists and patients (Wyniecki et al., 2011). Furthermore, anesthetic drugs have been shown to interact with antiretroviral drugs, such as benzodiazepines, opioids, and nonsteroidal anti-inflammatory drugs (Littlewood, 2008). Although the incidence rate of complications was slightly higher in the general anesthesia group, our results indicate that general anesthesia was reliable and safe, irrespective of the disease before the surgery status and surgical category. Some studies have also reported (Mathew et al., 2007) that there was no difference in general anesthetic management between patients with and without HIV-infection, which suggested that general anesthesia was appropriate for HIV-infected patients (Rodriguez-Merchan, 2006).

HIV infects vital cells in the human immune system such as $\mathrm{CD} 4^{+} \mathrm{T}$ cells, which leads to reduced levels of $\mathrm{CD}^{+} \mathrm{T}$ cells. When $\mathrm{CD} 4^{+} \mathrm{T}$ cell numbers decline below a critical level, cell-mediated immunity is compromised, and the body becomes progressively more susceptible to opportunistic infections (Nelson et al., 2009). In this study, patients with HIV infection had compromised immune function and some patients also suffered from other infections. Indeed, exogenous infection through intravertebral anesthesia was found to be a risk factor that accelerated the development of disease (Evron et al., 2004). However, our results indicated that intravertebral anesthesia did not increase the incidence rate of nervous system infection. None of patients in our study contracted a nervous system infection, which might be relevant to the neuronophagia characteristics of HIV.

A previous study found an increased incidence of fever in patients treated with general anesthesia or CSEA (Bremerich et al., 2003), and this result correlated with the severity of the primary disease and the complexity of the surgical procedure in those patients (Nadal et al., 1999). In addition, the level of CD4 ${ }^{+} \mathrm{T}$ cells was higher in the CSEA group than the other groups, which indicated that patients in the CSEA group had better immune function. However, infectious cases were found in all groups after surgery, which demonstrated that perioperative immune function had no influence on postoperative complications and incidence rate of infection. Thus, the type of surgery did not create more opportunity for infection (Bougaki et al., 2003). 
In conclusion, the three anesthetic approaches assessed in this study ensured the stability of perioperative indexes in HIV-infected patients and they did not cause postoperative complications. The exclusion of different patients with underlying diseases, operation complex degrees of influence of interference factors, our data indicate that the general, local, and CSEA anesthetic approaches are safe and effective in HIV patients.

\section{Conflicts of interest}

The authors declare no conflict of interest.

\section{REFERENCES}

Avidan MS, Groves P, Blott M, Welch J, et al. (2002). Low complication rate associated with cesarean section under spinal anesthesia for HIV-1-infected women on antiretroviral therapy. Anesthesiology 97: 320-324.

Ayers J, Howton MJ and Layon AJ (1993). Postoperative complications in patients with human immunodeficiency virus disease. Clinical data and a literature review. Chest 103: 1800-1807.

Bajwa SJ and Kulshrestha A (2013). The potential anesthetic threats, challenges and intensive care considerations in patients with HIV infection. J. Pharm. Bioallied Sci. 5: 10-16.

Bornard L, Blay M, Roger PM, Raucoules-Aimé M, et al. (2011). Anaesthesia for HIV-infected patients. Ann. Fr. Anesth. Reanim. 30: 501-511.

Bougaki M, Tomioka T and Hanaoka K (2003). Anesthetic management of a hemophilia A patient with HIV infection: a case report. Masui 52: 183-186.

Bremerich DH, Ahr A, Büchner S, Hingott H, et al. (2003). Anesthetic regimen for HIV positive parturients undergoing elective cesarean section. Anaesthesist 52: 1124-1131.

Evron S, Glezerman M, Harow E, Sadan O, et al. (2004). Human immunodeficiency virus: anesthetic and obstetric considerations. Anesth. Analg. 98: 503-511.

Ferrero S and Bentivoglio G (2003). Post-operative complications after caesarean section in HIV-infected women. Arch. Gynecol. Obstet. 268: 268-273.

Karpelowsky JS, Millar AJ, van der Graaf N, van Bogerijen G, et al. (2011). Outcome of HIV-exposed uninfected children undergoing surgery. BMC Pediatr. 11: 69.

Klein AA, Pozniak A and Pandit JJ (2014). Salami slicing or living off the fat? Justifying multiple publications from a single HIV dataset. Anaesthesia 69: 195-198.

Krugova LV, Vartanov VIa, Vartanova IV, Shakhovskaia IN, et al. (2010). Anaesthesia and intensive care characteristics in HIV-infected pregnant patients receiving the antiretroviral therapy. Anesteziol. Reanimatol. 6: 32-36.

Littlewood KE (2008). The immunocompromised adult patient and surgery. Best Pract. Res. Clin. Anaesthesiol. 22: 585609.

Lund AK, Lucero J, Herbert L, Liu Y, et al. (2011). Human immunodeficiency virus transgenic rats exhibit pulmonary hypertension. Am. J. Physiol. Lung Cell. Mol. Physiol. 301: L315-326.

Madzimbamuto FD, Ray S and Mogobe KD (2013). Integration of HIV care into maternal health services: a crucial change required in improving quality of obstetric care in countries with high HIV prevalence. BMC Int. Health Hum. Rights 13: 27.

Mathew J, Maddali MM and Fahr J (2007). Prolonged muscle weakness following general anesthesia in a parturient on combined antiretroviral therapy - a case report. Middle East J. Anesthesiol. 19: 673-678.

Nadal SR, Manzione CR, Horta SH and Galvão V (1999). Management of idiopathic ulcer of the anal canal by excision in HIV-positive patients. Dis. Colon. Rectum 42: 1598-1601.

Nelson L, Fried M and Stewart K (2009). HIV-infected patients: the risks of surgery. J. Perioper. Pract. 19: 24-30.

Redman LA, Naidoo P and Biccard BM (2014). HIV, vascular surgery and cardiovascular outcomes: a South African cohort study. Anaesthesia 69: 208-213.

Rodriguez-Merchan EC (2006). HIV and HCV coinfected haemophilia patients: what are the best options of orthopaedic treatment? Haemophilia 12: 90-101.

Wyniecki A, Zetlaoui P, Bruyère M and Benhamou D (2011). Bilateral catheter for continuous TAP block and postoperative pain relief after gynecologic surgery. Ann. Fr. Anesth. Reanim. 30: 67-69. 